\title{
Bi-Modality in the short-term photometric Properties of the Intermediate Polar V1062 Tau
}

\author{
Y. Lipkin, E. M. Leibowitz
}

School of physics and Astronomy and the Wise Observatory, Raymond and Beverly Sackler Faculty of Exact Sciences, Tel-Aviv University, Tel Aviv,69978, Israel; email: yiftah@wise.tau.ac.il, elia@wise.tau.ac.il

\section{Orio}

Istituto Nazionale di Astrofisica (INAF), Osservatorio Astronomico di Torino, Strada Osservatorio, 20, I-10025 Pino Torinese (TO), Italy; Department of Astronomy, $475 \mathrm{~N}$. Charter Street, University of Wisconsin, Madison,WI 53706,USA; email:orio@cow.physics.wisc.edu

\begin{abstract}
We conducted coordinated time-resolved observations of the long-period Intermediate Polar V1062 Tauri at the WIYN Observatory in Kitt Peak, Arizona and at Wise Observatory, Israel, and follow-up observations at the second site. We confirm the presence of two previously reported periodicities of the system: a long period $(\mathrm{P} \approx 10 \mathrm{~h})$ which was interpreted as the orbital period of the underlying binary system, and a shorter one $(P \approx 1 \mathrm{~h})$, which was interpreted as the spin of the white dwarf. Our data also revealed a third photometric periodicity, corresponding to the orbital sideband of the spin period (OSB). The amplitude of the OSB was found to be strongly correlated with the varying brightness of the system at the orbital cycle.

Our observations suggest bi-modality in the photometric characteristics of the star. In one mode, the light of V1062 Tau varies with the spin period of the white dwarf, whereas the OSB is undetected. In the other mode, the OSB is the main modulation at short time-scales, and the spin period is absent from the light curve. Switching between the two modes occurred three times during the 10 weeks that spanned our observations. Also, we detected an outburst of $1.1 \mathrm{mag}$, which lasted between $\sim 1$ and $\sim 5$ days. In addition to the outburst, secular variations in the brightness of the star ( $0.3 \mathrm{mag}$ on time scale of a few of tens of days) suggest that the system was in a brief low state during 2002 January.
\end{abstract}

\section{Introduction}

The Intermediate Polar (IP) V1062 Tauri (H0459+246) was discovered as an X-ray source by Remillard et al. (1994). The optical counterpart was identified as a $V \approx 16 \mathrm{mag}$ star with a strong UV flux. The optical spectrum of the star, as well as its X-ray and photometric features were characteristic of a magnetic CV (Remillard et al. 1994). 


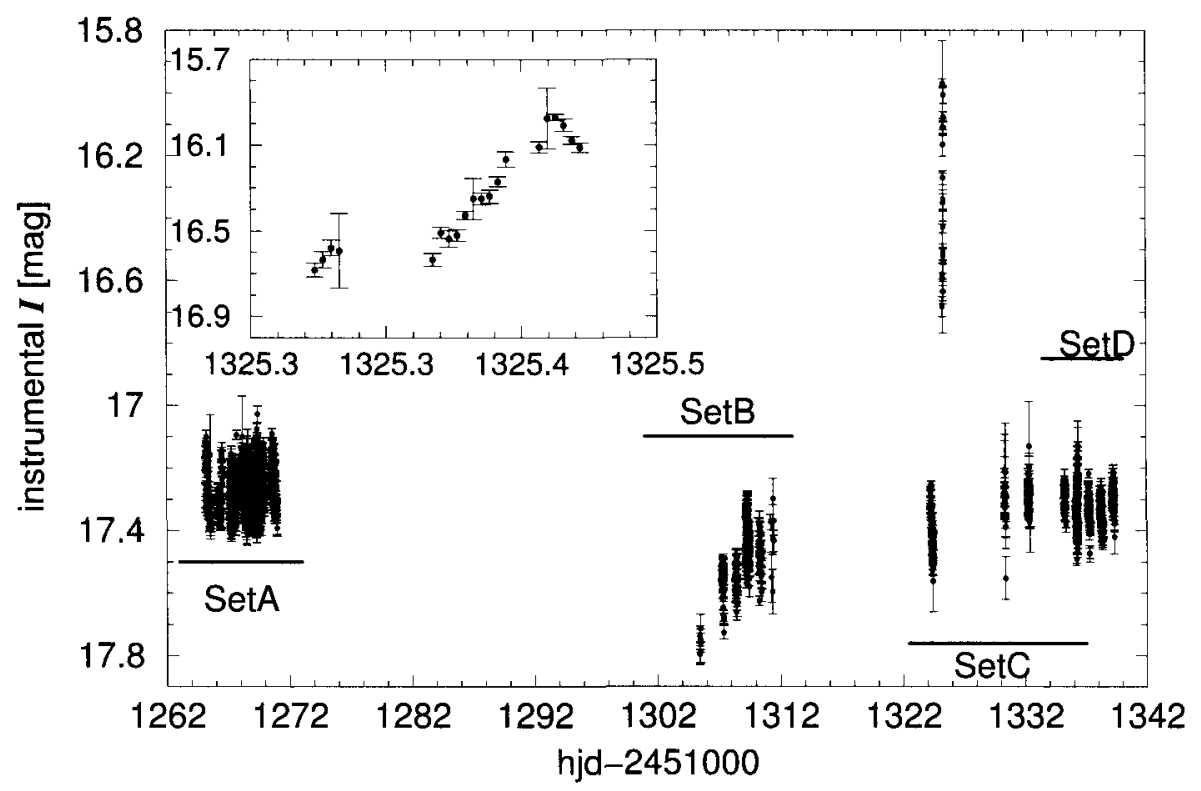

Figure 1. Light curve of V1062 Tau, 2001 Dec. - 2002 Mar. Inset: A blow-up of the outburst night, 2002 Feb 19

Remillard et al. (1994) found two periodicities in the light curve (LC) of the object. A long one $(P=9.952 \pm 0.074 \mathrm{~h})$, observable only in optical light, was interpreted as the orbital period of the underlying binary system. A shorter periodicity, which was detected both in optical $(P=63.2 \pm 0.3 \mathrm{~min})$, and in $\mathrm{X}$-ray data obtained with EXOSAT $(P=62.1 \pm 0.9 \mathrm{~min})$, was interpreted as the spin period of the magnetic white dwarf.

Hellier, Beardmore, \& Mukai (2002) confirmed the presence of the short periodicity in combined X-ray data from ASCA and RXTE, and refined its period to $61.73 \pm 0.13 \mathrm{~min}$

Here we report on main results of a photometry campaign on V1062 Tau, which we have performed in 2001/02. A more complete account of the results and an analysis of them is in preparation.

\section{Observations and Analysis}

We conducted time-resolved photometry of V1062 Tau during 5 nights in 2001 Dec., with the $1 \mathrm{~m}$ telescope of Wise Observatory, Israel (WO), and the $0.9 \mathrm{~m}$ telescope of the WIYN observatory at Kitt Peak, Arizona. Additional 15 nights of photometry were conducted from WO only, in 2002 Feb. - 2002 Mar. Observations were conducted primarily through $I$ filter, with a typical exposure time of $300 \mathrm{sec}$. 
Data reduction was carried out in the standard procedure followed in WO (see e.g. Lipkin et al. 2001). The light curve accumulated in our program is shown in Fig. 1.

\section{Results}

\subsection{Secular variations}

Low State On 2002 Jan 30 V1062 Tau was observed 0.3 mag fainter than the preceding observations, 34 days before. Over the following $\sim 50$ nights of observations, the star brightened, and nearly returned to its original brightness state (Fig. 1). This suggests a possible brief low state of V1062 Tau during 2002 January.

Short outburst On 2002 Feb 19 the star was observed in outburst, which lasted between $\sim 1$ and $\sim 6$ days, during which the brightness of the star increased by at least a factor of three (Fig. 1).

The last four data points of the outburst LC exhibit a declining trend, which is not in phase with the orbital, nor with the spin variation (Fig. 1, inset). If this marks the beginning of the decline from outburst maximum, then this is a short ( $\Delta T_{\text {outburst }} \approx 1-2$ days), low amplitude $(\Delta I=1.16 \mathrm{mag})$ outburst. Similar short and weak outbursts were observed in only two other $I P_{\mathrm{S}}$ : V1223 Sgr (only one observed outburst, van Amerongen \& van Paradijs, 1989), and TV Col (a few short outbursts, e.g. Hellier \& Buckley, 1993).

\subsection{Periodicities}

The power spectrum (PS, Scargle 1982) of our entire data set is dominated by the fundamental and the first harmonic of the orbital frequency $(\Omega)$, and by the fundamental harmonics of the spin frequency $(\omega)$ and the OSB ( $\omega-\Omega$, Fig. 2). The first harmonics of $\omega$ and $\omega-\Omega$ are also detectable (not shown).

The best fit values we derived for the three periods are: $P_{\text {orb }}=9.9082 \pm$ $0.0006 \mathrm{~h}, P_{\text {spin }}=61.43 \pm 0.05 \mathrm{~m}$, and $P_{O S B}=68.45 \pm 0.09 \mathrm{~m}$ (errors are $1-\sigma$ confidence levels, derived using the boot-strap method, Efron \& Tibshirani 1993). The orbital period we derived agrees with the one measured by Remillard et al. (1994). The value we obtained for the spin agrees with that of Hellier et al. (2002), and with the value Remillard et al. (1994) measured in X-ray, but significantly differs from the optical measurement given by the latter $(P=$ $63.2 \pm 0.3 \mathrm{~m}$ ). However, an examination of Fig. 11 of Remillard et al. (1994), showing a variance statistics of their $I$-band data, suggests that the trough selected by the authors as the spin period is probably an alias. The two adjacent troughs, corresponding to $\sim 61.0 \mathrm{~m}$ and $\sim 66.7 \mathrm{~m}$, probably represent the existence of both the spin, and the OSB in their data. Another distinct trough, at $\sim 31 \mathrm{~m}$ (corresponding to $2 \omega$ ), supports this alternative selection.

\subsection{Period Evolution}

Fig. 3 (left panel) shows the residual PS of a set of 6 nights between $2001 \mathrm{Dec}$ 21 - Dec 26 (hereafter SetA, Fig. 1), after removing the signal of $P_{o r b}$, from the LC. The dominant signal in the residual PS is $\omega-\Omega$. No appreciable power is 


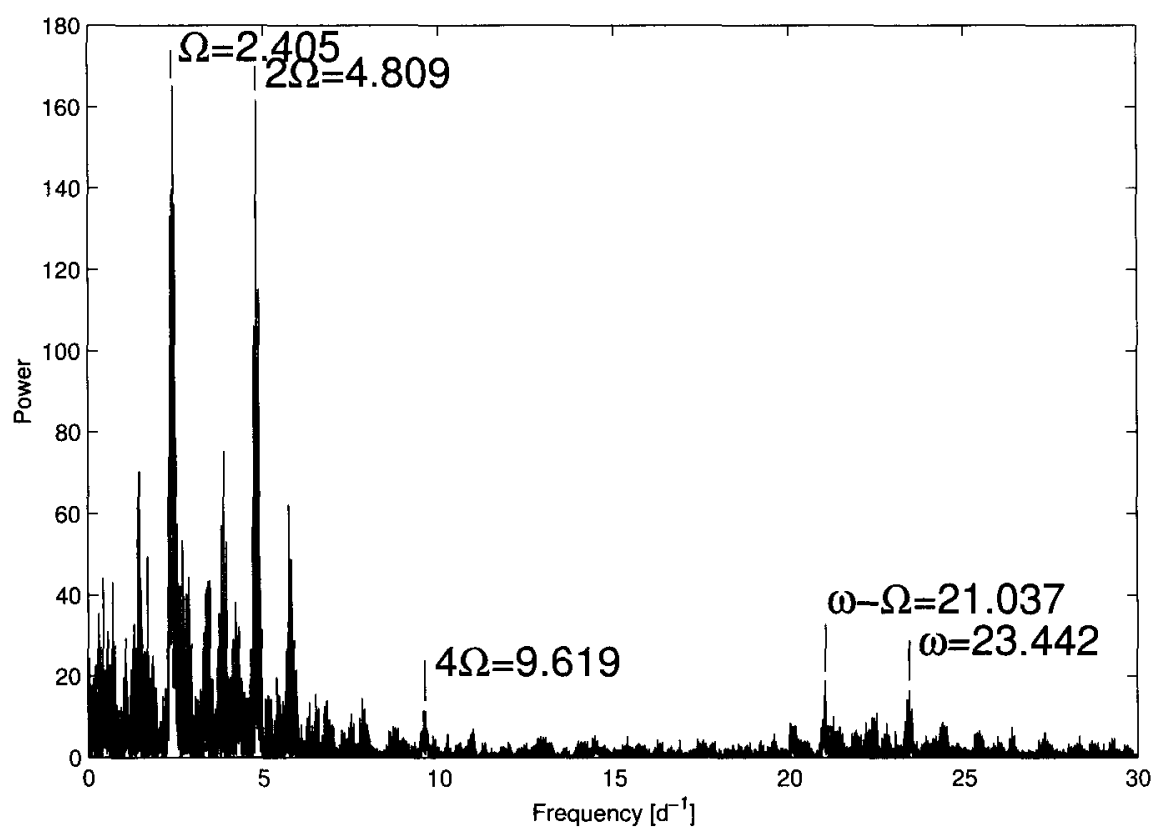

Figure 2. Power spectrum of V0162 Tau, 2001 Dec-2002 Mar. An outburst night ( $\mathrm{HJD}=2452325)$ was omitted from the analysis. Secular variations in the data were eliminated prior to the analysis by subtracting the average from the 2001 December dataset, a parabola from the 2002 February dataset, and the average from the 2002 March dataset.

found in the spin frequency. The calculated upper limit for the semi-amplitude of $P_{\text {spin }}$ in this set is $0.005 \mathrm{mag}$.

A $\chi^{2}$-periodogram of a group of 6 nights between 2002 Jan $30-$ Feb 05 (SetB) shows different results (Fig. 3, right panel). Here, the spin period is the dominant signal, and there is no significant signal af $\omega-\Omega$ (with an upper limit of $0.005 \mathrm{mag}$ for the semi-amplitude). Similar to SetA, in a third subset (SetC: 2002 Feb 18 - Mar 02, 5 nights), the main signal is the OSB frequency, and $\omega$ is marginal in the PS (Fig. 4, left panel). Finally, the fourth subset of out data (SetD: 2002 Mar 01 - Mar 05, 5 nights, is modulated by the spin period, but not by $\omega-\Omega$ (Fig. 4, right panel, note a two-day overlap with SetC).

Period analysis of the overlapping SetC and SetD suggests that transition between a mode in which the star's light was modulated by $\omega$ to a mode in which $\omega-\Omega$ moulted the object's LC, occurred within a time span of two days.

\subsection{Waveforms and Amplitudes}

The waveform of the orbital modulation, derived from the $\mathrm{LC}$ of Set $\mathrm{A}$ is shown in Fig. 5. The double-humped shape of the signal is somewhat different from the one shown in Remillard et al. (1994), with a more pronounced secondary minimum, and a greater asymmetry between the primary and secondary max- 

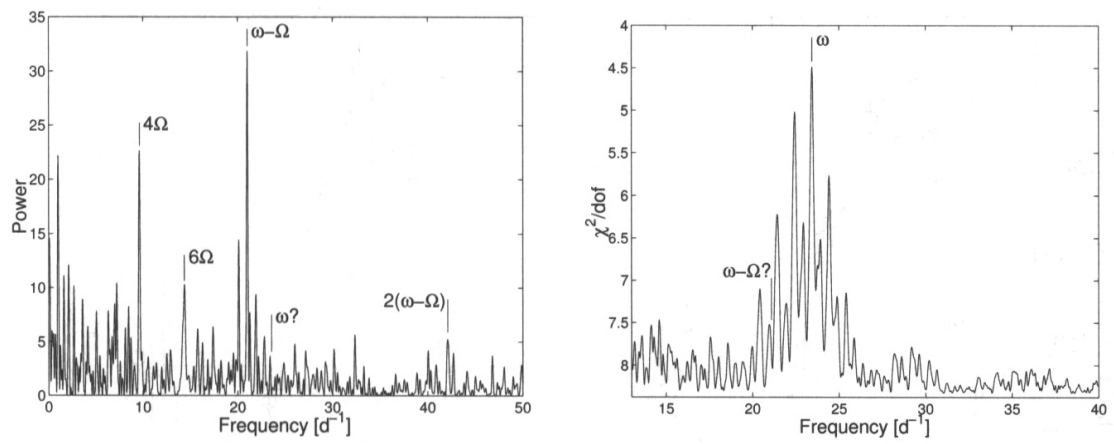

Figure 3. Left panel: a PS of 9 nights between 2001 Dec 21 and 2001 Dec 26 (SetA). The signal of $P_{\text {orb }}$ was pre-subtracted Right Panel: a $\chi^{2}$-periodogram of of 6 nights between 2002 Jan 30 2992 Feb 05 (SetB).
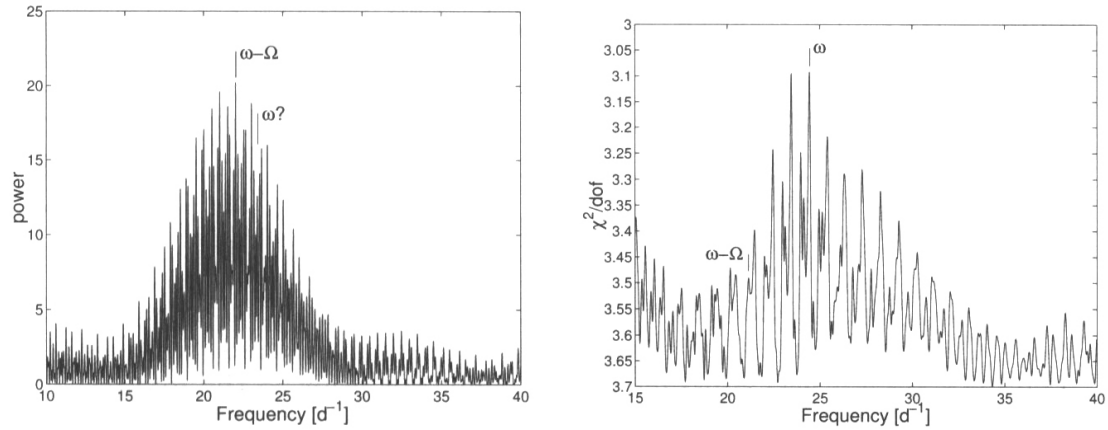

Figure 4. Left panel: a PS of 5 nights between 2002 Feb 18 - Mar 02 (setB). Right Panel: a $\chi^{2}$-periodogram of of 5 nights between 2002 Mar 01 - Mar 05 (setB). Note that SetC and SetD overlap.

ima. The amplitude of the orbital variation is $\Delta I=0.191 \pm 0.013 \mathrm{mag}$. Other subsets of our data yield compatible waveforms for $P_{\text {orb }}$

The waveform of the spin, measured in setB and SetD is shown in Fig. 6, left panel (upper and lower graphs, are the waveform in setB and SetD, respectively). The spin waveform differs in the two data sets, both in shape and in amplitude (in setB $\Delta I=0.18 \pm 0.02 \mathrm{mag}$, and in SetD $\Delta I=0.09 \pm 0.03 \mathrm{mag}$ ).

Simillarly, SetA and SetC yielded different waveforms for the OSB signal (Fig. 6, right panel. The amplitudes are: $\Delta I=0.064 \pm 0.011 \mathrm{mag}$, and $\Delta I=$ $0.11 \pm 0.03 \mathrm{mag}$, in SetA and SetC, respectively).

We further measured the amplitudes of the OSB at various orbital phases. In SetA, the OSB amplitude was strongly correlated with the the varying brightness of the system at the orbital cycle.

Furthermore, the amplitudes of both short periods were found to vary over different orbital phases, however, the magnitude and form of these correlations were different in the various data subsets. 


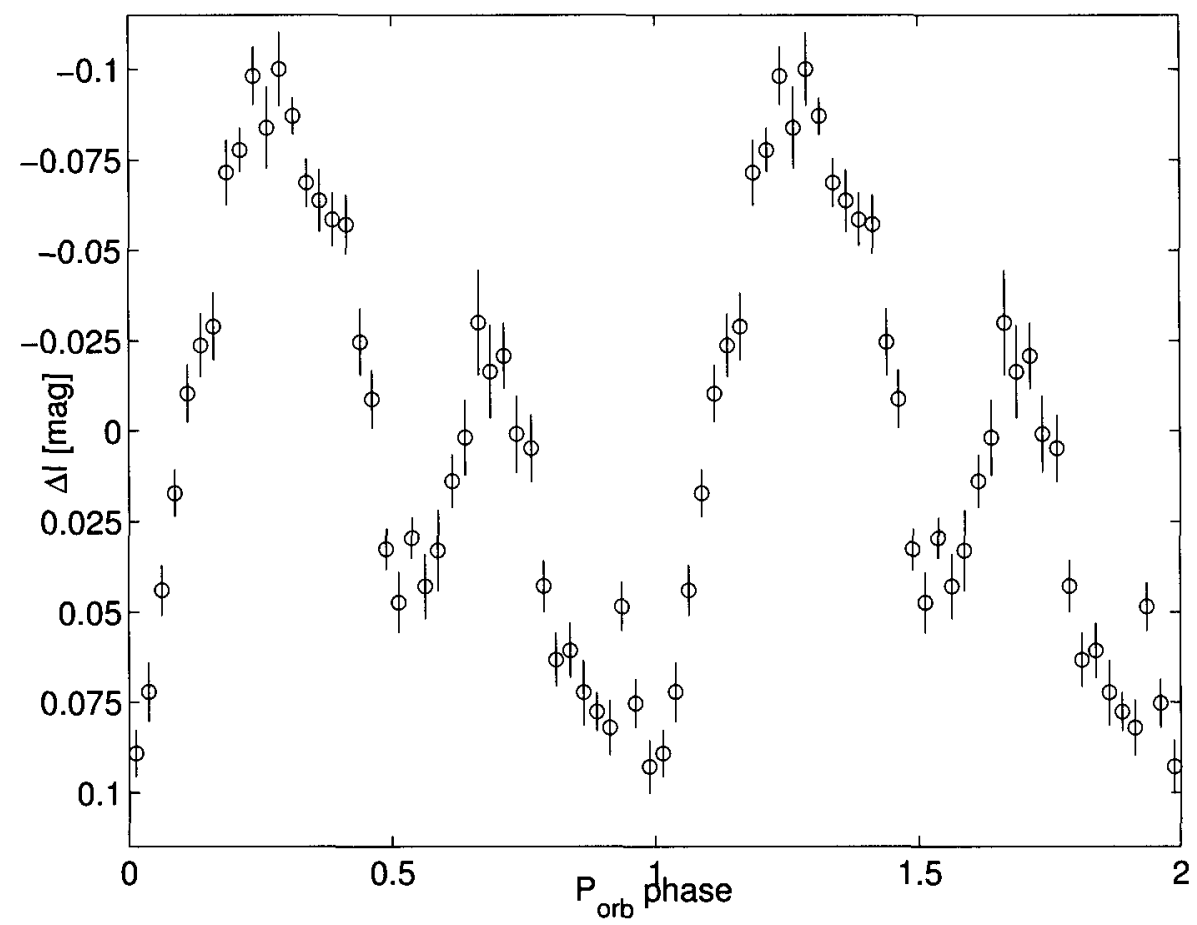

Figure 5. SetA, folded over $P_{\text {orb }}$, and averaged in 40 evenly spaced bins. The error bars are the standard deviation measured in each bin. The OSB period was subtracted from the data prior to the folding.

\section{Summary}

1. We detected an outburst of V1062 Tau, which occurred on a time scale of $\sim 1$ day to no more than 6 days. If the outburst was a short one, then V1062 Tau would be the third known IP to harbor such short outbursts.

2. We detected a possible brief low state of V1062 Tau, a few days to one month before its outburst.

3. During our observations, V1062 Tau oscillated between two states: one in which the light of the star is modulated by the spin period, and another one in which the OSB modulates the object's brightness. Our analysis suggest that transition between the two modes occurs on a time scale of two days.

4. The amplitudes of both the spin and the OSB were found to be correlated with the orbital phase. The form of the correlation varied in different subsets of our data.

Further analysis and possible interpretations will be published elsewhere. 

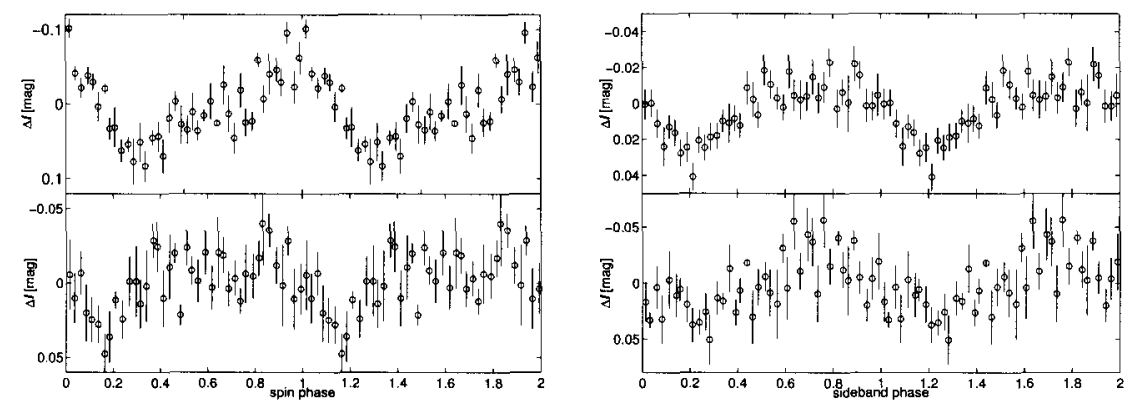

Figure 6. Left panel: the spin waveform in setB (upper graph), and SetD (lower graph)

Right panel: the OSB waveform in SetA (upper graph), and SetC (lower graph)

\section{Acknowledgments}

YL acknowledges partial financial support received from IAU to take part in the IAU Colloquium on MCVs.

\section{References}

Efron, B. \& Tibshirani, R. J. 1993, An Introduction to the Bootstrap, Chapman $\&$ Hall

Hellier, C., Beardmore, A. P., \& Mukai, K., 2002, A\&A, 389,

Hellier, C. \& Buckley D.A.H. 1993, MNRAS, 265, 766

Lipkin, Y., Leibowitz, E.M., Retter, A., \& Shemmer, O. 2001, MNRAS, 328 , 1169

Munari, U., Zwitter, T., \& Bragaglia, A. 1997 A\&AS. 122, 495

Remillard, R.A., Bradt, H.V., Brissenden, R.J.V., Buckley, D.A.H., Schwartz, D.A., Silber, A., Stroozas, B.A., \& Tuohy, I.R. 1994, ApJ, 428, 785

van Amerongen, S. \& van Paradijs, J. 1998, A\&A, 1989, 219, 195

Scargle J. D. 1982, ApJ, 263, 835 